\title{
Effects of (MHD) and wall properties oscillatory flow for williamson fluid with constant viscosity through porous channel
}

\author{
https://doi.org/10.31185/wjcm.Vol1.Iss1.12 \\ Zain Alabdeen A.N.ALSAFI ( $\left.{ }^{\square}\right)$ \\ College of Computer Science and Information Technology Wasit University \\ leccit3@uowasit.edu.iq \\ Ahmed A.H. Al-Aridhee \\ Department of Mathematics, College of Science, University of Al-Qadisiyah, \\ Diwaniya, Iraq \\ ahmedalardy845egmail.com \\ Saif Razzaq Al-Waily \\ Department of Mathematics, College of Science, University of Al-Qadisiyah, \\ Diwaniya, Iraq \\ ma.post20@qu.edu.iq
}

\begin{abstract}
In this research, the williamson flow with heat transfer through the tube of compliant wall properties with slip at boundaries is analyzed analytically. An approximated theoretical model is constructed of springbacked flexible compliant walls pipe, chosen to move as sinusoidal wave
\end{abstract}

Keywords-Wall properties, Heat transfer, williamson flow

\section{Introduction}

The flow of electrically oriented fluid has a lot of applications and this science deal with many branches. In astronomy, it helps to understand what happens in the sun, such as rotating solar spots, what happens inside other stars through their life cycle, and geology. To reduce it in an area far from the walls of the container by magnetic fields, so that the temperature and pressure can be increased to values close to the corresponding values within the stars and so on. Nigam and Singh [1], have studied the effect of heat-transfer on laminar flow among parallel flakes under the impact of transverse magnetic field. Attia and Kotb [2], have studied the heat-transfer with MHD flow of viscous fluid among two parallel flakes. The hydro-magnetic free convection flow during a porous medium among two parallel plates was discussed by Massias et al. [3]. Mustafa [4], Researches relevant to this type are presented in the following. Pandey and Chaube [5] investigated the influence of flexible wall features on the couple stress fluid travelling peristaltically. Hina et al. [6] investigation handled the wall properties impact on 
the wavy flow of Maxwellian liquid through duct. Radhakrishnamacharya and Srinivasulu [7] took both effects of wall properties and heat transfer on the peristaltic transport of fluid via channel in their study. Srinivas et al. [8] studied the influence of several variables of wall slip, magnetic field, wall properties, and heat transfer on the wavy flow through channel. Also Srinivas et al.

\section{Mathematical Formulation:}

Let us consider the flow of a Williamson fluid in a channel of width $h$ under the effects of electrically applied magnetic field and radioactive heat transfer as depicted in (Fig. 2.1). Supposed that the fluid has very small electromagnetic force produced and the electrical conductivity is small. We are considering Cartesian coordinate system such that, $(\mathrm{u}(\mathrm{y}), 0,0)$ is a velocity vector in which $\mathrm{u}$ is the $\mathrm{x}$-component of velocity and $\mathrm{y}$ is perpendicular to the $\mathrm{x}$-axis.
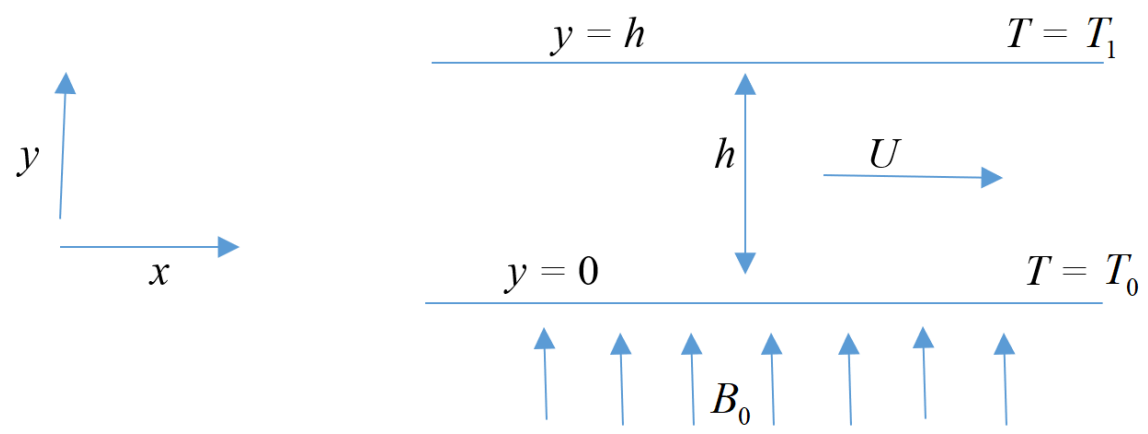

Fig. 1. Physical model

\section{Basic Equations:}

The basic equations governing for Williamson fluid are given by:

The continuity equation is given by:

$\frac{\partial \bar{u}}{\partial \bar{x}}+\frac{\partial \bar{v}}{\partial \bar{y}}=0$

The momentum equations are:

In the $x$ - direction:

$\rho\left(\frac{\partial \bar{u}}{\partial \bar{t}}+\bar{u} \frac{\partial \bar{u}}{\partial \bar{x}}+\bar{v} \frac{\partial \bar{u}}{\partial \bar{y}}\right)=-\frac{\partial \bar{p}}{\partial \bar{x}}+\frac{\partial \bar{\tau}_{\overline{x x}}}{\partial \bar{x}}+\frac{\partial \bar{\tau}_{\overline{x y}}}{\partial \bar{y}}+\rho g \beta\left(T-T_{0}\right)-\sigma B_{0}^{2} \bar{u}-\frac{\mu_{0}}{k} \bar{u}$

In the $y$-direction:

$\rho\left(\frac{\partial \bar{v}}{\partial \bar{t}}+\bar{u} \frac{\partial \bar{v}}{\partial \bar{x}}+\bar{v} \frac{\partial \bar{v}}{\partial \bar{y}}\right)=-\frac{\partial \bar{p}}{\partial \bar{y}}+\frac{\partial \bar{\tau}_{\overline{x y}}}{\partial \bar{x}}+\frac{\partial \bar{\tau}_{\overline{y y}}}{\partial \bar{y}}-\frac{\mu_{0}}{k} \bar{v}$

The temperature equation is given by:

$\rho \frac{\partial T}{\partial \bar{t}}=\frac{K}{C_{p}} \frac{\partial^{2} T}{\partial \bar{y}^{2}}-\frac{1}{C_{p}} \frac{\partial q}{\partial y}$ 
where $\bar{u}$ is the axial velocity, $T$ is a fluid temperature, $B_{0}$ is a magnetic field strength, $\rho$ is a fluid density, $\sigma$ is a conductivity of the fluid, $\beta$ is a coefficient of volume amplification due to temperature, $g$ is a hastening due to gravity, $\mathrm{k}$ is a permeability, $c_{p}$ is a specific heat at constant pressure, $K$ is a thermal conductivity and $q$ is a radioactive heat flux.

The temperatures at the walls of the channel are given as:

$T=T_{0}$ at $\bar{y}=0$, and $T=T_{1}$ at $\bar{y}=h$

The radioactive heat flux [9] is given by:

$\frac{\partial q}{\partial y}=4 \eta^{2}\left(T_{0}-T\right)$

The radiation absorption denoted by $\eta$.

\section{$4 \quad$ Flexibe Wall}

The governing equation of motion of the flexible wall may be expressed as:

$L^{*}=\bar{P}-\bar{p}_{0}$

where $L^{*}$ is an operator, which is used to represent the motion of stretched membrane with viscosity damping forces such that

$L^{*}=-k \frac{\partial^{2}}{\partial \bar{x}^{2}}+m_{1} \frac{\partial^{2}}{\partial \bar{t}^{2}}+C \frac{\partial}{\partial \bar{t}}$

where $k$ is the eiastic tension in the membrane, $m_{1}$ is the mass per unit area, $C$ is the coefficient of viscous damping forces.

The continuity of stress at $\bar{r}=\bar{h}$ and using momentum equation, yield:

$\frac{\partial}{\partial \bar{x}} L^{*}(\bar{h})=\frac{\partial \bar{p}}{\partial \bar{x}}=-\rho\left(\frac{\partial \bar{u}}{\partial \bar{t}}+\bar{u} \frac{\partial \bar{u}}{\partial \bar{x}}+\bar{v} \frac{\partial \bar{u}}{\partial \bar{y}}\right)+\frac{\partial \bar{\tau}_{\overline{x x}}}{\partial \bar{x}}+\frac{\partial \bar{\tau}_{\overline{x y}}}{\partial \bar{y}}+\rho g \beta\left(T-T_{0}\right)-\sigma B_{0}^{2} \bar{u}-$

$\frac{\mu_{0}}{k} \bar{u}$

\section{The Fundamental Equation:}

The fundamental equation for Williamson fluid given by:

$\boldsymbol{S}=-\bar{p} \boldsymbol{I}+\tau$

$\bar{\tau}=\left[\mu_{\infty}+\left(\mu_{0}-\mu_{\infty}\right)(1+\Gamma \overline{\dot{\gamma}})^{-1}\right] A_{1}$

where $\bar{p}$ is the pressure, $\boldsymbol{I}$ is the unit tensor, $\bar{\tau}$ is the extra stress tensor, $\Gamma$ is the time constant, $\mu_{\infty}$ and $\mu_{0}$ are the infinite shear rate viscosity and zero shear rate viscosity, then $\dot{\gamma}$ is defined as:

$$
\dot{\gamma}=\sqrt{\frac{1}{2} \sum_{i} \sum_{j} \dot{\gamma}_{i j} \dot{\gamma}_{j i}}=\sqrt{\frac{1}{2} \Pi}
$$

Here $\prod$ is the second invariant strain tensor. We consider the fundamental Eq. (11), the case for which $\Gamma \dot{\gamma}<1$, and $\mu_{\infty}=0$. We can write the component of extra stress tensor according to follows as: 


$$
\bar{\tau}=\mu_{0}[(1+\Gamma \bar{\gamma})] \mathrm{A}_{1}
$$

Then

$$
\begin{aligned}
& \bar{\tau}_{\overline{x x}}=\bar{\tau}_{\overline{y y}}=\bar{\tau}_{\overline{x z}}=\bar{\tau}_{\overline{y z}}=0 \\
& \bar{\tau}_{\overline{x y}}=\bar{\tau}_{\overline{y x}}=\mu_{0}[(1+\Gamma \bar{\gamma})]\left(\bar{u}_{y}+\bar{v}_{x}\right)=\mu_{0}[(1+\Gamma \bar{\gamma})]\left(\bar{u}_{y}\right)
\end{aligned}
$$

\section{Method of Solution:}

The governing equations of the motion, we may introduce the non-dimensional conditions are as follows:

$$
\begin{gathered}
x=\frac{\bar{x}}{h}, y=\frac{\bar{y}}{h}, u=\frac{\bar{u}}{U}, \theta=\frac{T-T_{0}}{T_{1}-T_{0}}, t=\frac{\bar{t} U}{h}, p=\frac{\bar{p} h}{\mu U}, M^{2}=\frac{\sigma B_{0}^{2} h^{2}}{\mu} \\
W e=\frac{\Gamma U}{h}, \tau_{x x}=\frac{h}{\mu_{0} U} \bar{\tau}_{\overline{x x}}, \tau_{x y}=\frac{h}{\mu_{0} U} \bar{\tau}_{\overline{x y}}, \dot{\gamma}=\frac{h}{U} \bar{\gamma}, D a=\frac{k}{h^{2}} \\
R e=\frac{\rho h U}{\mu}, P e=\frac{\rho h U c_{p}}{K}, N^{2}=\frac{4 \eta^{2} h^{2}}{K}, G r=\frac{\rho g \beta h^{2}\left(T_{1}-T_{0}\right)}{\mu U}
\end{gathered}
$$

where $(U)$ is the mean flow velocity, $(D a)$ is Darcy number, $(R e)$ is Reynolds number, $(\mathrm{Gr})$ is Grashof number, $(M)$ is magnetic parameter, $(P e)$ is the Peclet number and $(N)$ is the radiation parameter.

Substituting the Eq. (16) into the Eq. (1-4), we have

$$
\frac{\partial u}{\partial x}+\frac{\partial v}{\partial y}=0
$$

$$
R e \frac{\partial u}{\partial t}=-\frac{\partial p}{\partial x}+\frac{\partial}{\partial y}\left[\frac{\partial u}{\partial y}+w e\left(\frac{\partial u}{\partial y}\right)^{2}\right]+\operatorname{Gr} \theta-\left(M^{2}+\frac{1}{D a}\right) u
$$

$P e \frac{\partial \theta}{\partial t}=\frac{\partial^{2} \theta}{\partial y^{2}}+N^{2} \theta$

$-\frac{k a}{\mu_{0} U h} \frac{\partial^{3}(h)}{\partial x^{3}}+\frac{m_{1} a}{\mu_{0} U h} \frac{\partial^{3}(h)}{\partial t^{2} \partial x}+\frac{h C a}{\mu_{0} U} \frac{\partial^{2}(h)}{\partial t \partial x}=-R e \frac{\partial u}{\partial t}+\frac{\partial}{\partial y}\left[\frac{\partial u}{\partial y}+w e\left(\frac{\partial u}{\partial y}\right)^{2}\right]+\operatorname{Gr} \theta-$

$\left(M^{2}+\frac{1}{D a}\right) u$

$-F_{1} \frac{\partial^{3}(h)}{\partial x^{3}}+F_{2} \frac{\partial^{3}(h)}{\partial t^{2} \partial x}+F_{3} \frac{\partial^{2}(h)}{\partial t \partial x}=-R e \frac{\partial u}{\partial t}+\frac{\partial}{\partial y}\left[\frac{\partial u}{\partial y}+w e\left(\frac{\partial u}{\partial y}\right)^{2}\right]+\operatorname{Gr} \theta-\left(M^{2}+\frac{1}{D a}\right) u$

$$
F_{1}=\frac{k a}{\mu_{0} U h}, F_{2}=\frac{m_{1} a}{\mu_{0} U h}, F_{3}=\frac{h C a}{\mu_{0} U}
$$

Now, we solve the temperature Eq. (19) with boundary conditions $\theta(0)=0, \theta(1)=1$

Let

$\theta(y, t)=\theta_{0}(y) e^{i \omega t}$

The frequency of the oscillation denoted by $\omega$.

Substituting the Eq. (23) into the Eq. (19), we have 


$$
\begin{gathered}
P e \frac{\partial}{\partial t}\left(\theta_{0}(y) e^{i \omega t}\right)=\frac{\partial^{2}}{\partial y^{2}}\left(\theta_{0}(y) e^{i \omega t}\right)+N^{2}\left(\theta_{0}(y) e^{i \omega t}\right) \\
\frac{\partial^{2} \theta_{0}}{\partial y^{2}}+\left(N^{2}-i \omega P e\right) \theta_{0}=0
\end{gathered}
$$

The solution of Eq. (24), is:

$$
\theta_{0}(y)=\csc (\varphi) \sin (\varphi y)
$$

where $\varphi=\sqrt{N^{2}-i \omega P e}$.

Hence

$$
\theta(y, t)=\csc (\varphi) \sin (\varphi y) e^{i \omega t}
$$

The solution of Eq. (21), is:

$$
u=B / A+e^{\sqrt{A} y}\left\{-\left(B /\left(A\left(1+e^{\sqrt{A}}\right)\right)\right)\right\}+e^{-\sqrt{A} y}\left\{-\left(\left(B e^{\sqrt{A}}\right) /\left(A \left(1+e^{\sqrt{A}}\right.\right.\right.\right.
$$

)$))\}+S *\left(1 / A\left\{-\left(\left(B e^{\sqrt{A} y}\right) /\left(\sqrt{A}\left(1+e^{\sqrt{A}}\right)\right)\right)+\left(B e^{\sqrt{A}-\sqrt{A} y}\right) /(\sqrt{A}\right.\right.$ $\left.\left.\left.e^{\sqrt{A}}\right)\right)\right\}\left\{-\left(\left(B e^{\sqrt{A} y}\right) /\left(1+e^{\sqrt{A}}\right)\right)-\left(B e^{\sqrt{A}-\sqrt{A} y}\right) /\left(1+e^{\sqrt{A}}\right)\right\} 2+e^{\sqrt{A} y}$

$$
\left(-\left(\left(\left\{-\left(\left(B e^{\sqrt{A} y}\right) /()^{\sqrt{A}}\left(1+e^{\sqrt{A}}\right)\right)\right)+\left(B e^{\sqrt{A}-\sqrt{A} y}\right) /\left(\sqrt { A } \left(1+e^{\sqrt{A}}\right.\right.\right.\right.\right.
$$

)$\left.)\}\left\{-\left(\left(B \mathbf{e}^{\sqrt{A} y}\right) /\left(1+e^{\sqrt{A}}\right)\right)-\left(B e^{\sqrt{A}-\sqrt{A} y}\right) /\left(1+e^{\sqrt{A}}\right)\right\} 2\right) /\left(A\left(1+e^{\sqrt{A}}\right.\right.$

)$))+e^{-\sqrt{A} y}\left(-1 /\left(A\left(1+e^{\sqrt{A}}\right)\right) e^{\sqrt{A}}\left\{-\left(\left(B e^{\sqrt{A} y}\right) /\left(\sqrt{A}\left(1+e^{\sqrt{A}}\right)\right)\right)+(B\right.\right.$ $\left.\left.e^{\sqrt{A}-\sqrt{A} y}\right) /\left(\sqrt{A}\left(1+e^{\sqrt{A}}\right)\right)\right\}\left\{-\left(\left(B^{e^{\sqrt{A} y}}\right) /\left(1+e^{\sqrt{A}}\right)\right)-\left(B^{e^{\sqrt{A}-\sqrt{A} y}}\right) /(1+\right.$ $\left.\left.\left.e^{\sqrt{A}}\right)\right\} 2\right)$ )

Where

$$
\begin{aligned}
& B=G r *(\operatorname{Csc}[\sqrt{\mathbf{k}}]) \operatorname{Sin}[\sqrt{\mathbf{k}} y] \\
& A=M 2+R e+1 / D a
\end{aligned}
$$

\section{Results and Discussion:}

We discuss the influence of heat transfer on the Magneto hydrodynamics oscillatory flow of Williamson fluid through a porous medium. Numerical assessments of analytical results and some of the graphically significant results are presented in figures (212). We use the (MATHEMATICA-12) program to find the numerical results and illustrations. The momentum equation is resolved by using perturbation technique and all the results are discussed graphically.

The velocity profile of Poiseuille flow is shown in figures (2-9). (Fig.2) shows the velocity profile $u$ decreases with the increasing $S$. (Fig.3) illustrates the influence $G r$ on the velocity profiles function $u$ vs. $y$. It is found by the increasing $G r$ the velocity 
profiles function $u$ increase. (Fig.4) shows that velocity profile $u$ rising up by the increasing influence the parameter $D a$. (Fig.5) shows that velocity profiles decreases with the increase of the parameters $P e$. (Fig.6) shows that velocity profiles decreases with the increase of the parameters $R e$. (Fig.7) shows the velocity profile $u$ increases by the increasing $M$. (Fig.8) shows that velocity profiles decreases with the increase of the parameters $W$. (Fig. 9) shows that velocity profiles increasing with the increase of the parameters $N$.

Based on equation (26), (Fig.10) show that influence of $N$ on the temperature function $\theta$. The temperature increases with the increase in $N$. (Fig.11 we observed that the influence $P e$ in temperature $\theta$ by the increasing $P e$ then $\theta$ increases. (Fig.12 show us that with the increasing of $\omega$ the temperature $\theta$ decreases.

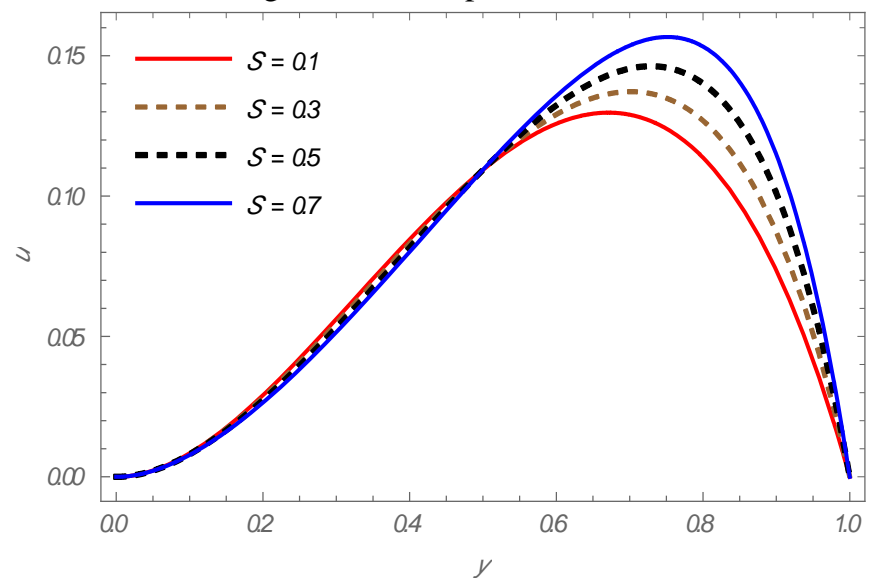

Fig. 2. Velocity profile for $S$ with $D a=1, M=1, \mathrm{~W}=1, \lambda=1, \operatorname{Re}=1, \mathrm{~N}=1.25, M=$ $1, P e=2, G r=2, t=0.5$.

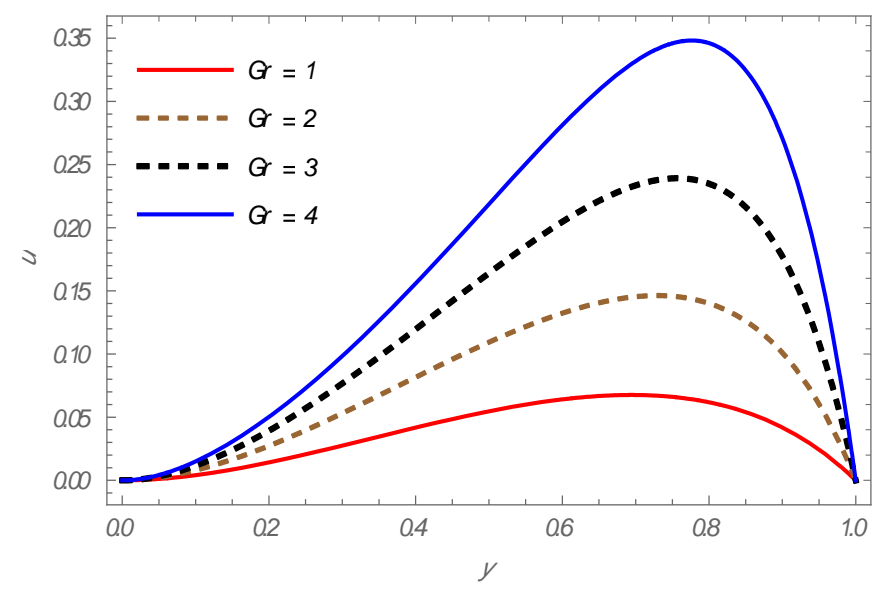

Fig. 3. Velocity profile for $\boldsymbol{G r}$ with $D a=1, M=1, \mathrm{~W}=1, \lambda=1, \mathrm{Re}=1, \mathrm{~N}=1.25, M=$ $1, P e=2, S=0.5, t=0.5$ 


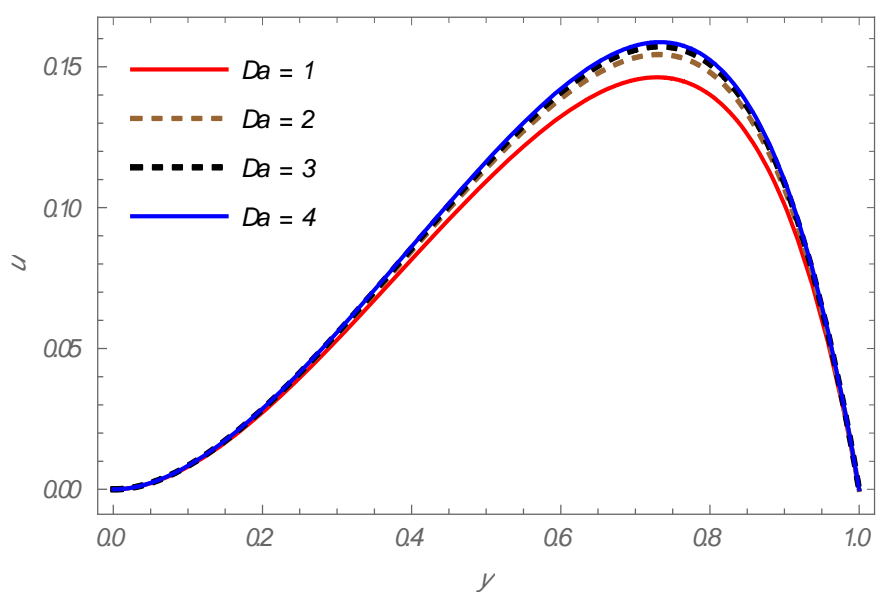

Fig. 4. Velocity profile for $\boldsymbol{D a}$ with $M=1, \mathrm{~W}=1, \lambda=1, \mathrm{Re}=1, \mathrm{~N}=1.25, M=1, P e=$ $2, G r=2, S=0.5, t=0.5$.

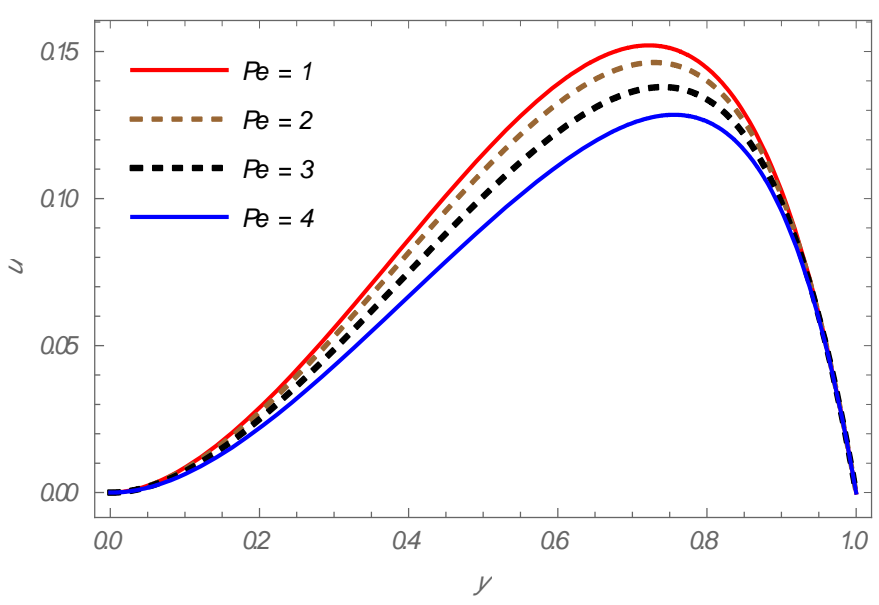

Fig. 5. Velocity profile for $\boldsymbol{P e}$ with $D a=1, M=1, \mathrm{~W}=1, \lambda=1, \operatorname{Re}=1, \mathrm{~N}=1.25, M=$ $1, G r=2, S=0.5, t=0.5$. 


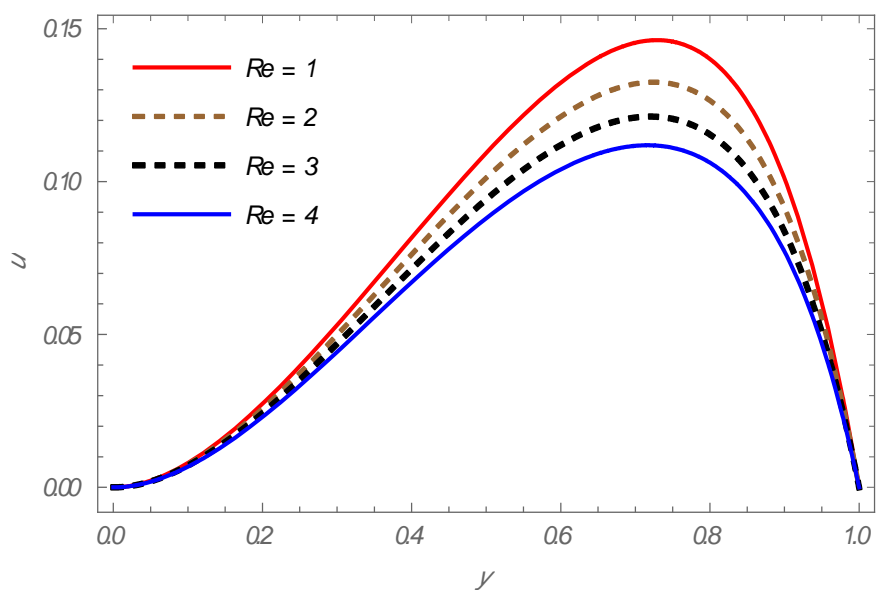

Fig. 6. Velocity profile for $\boldsymbol{R} \boldsymbol{e}$ with $D a=1, M=1, \mathrm{~W}=1, \lambda=1, \mathrm{~N}=1.25, M=1, P e=$ $2, G r=2, S=0.5, t=0.5$.

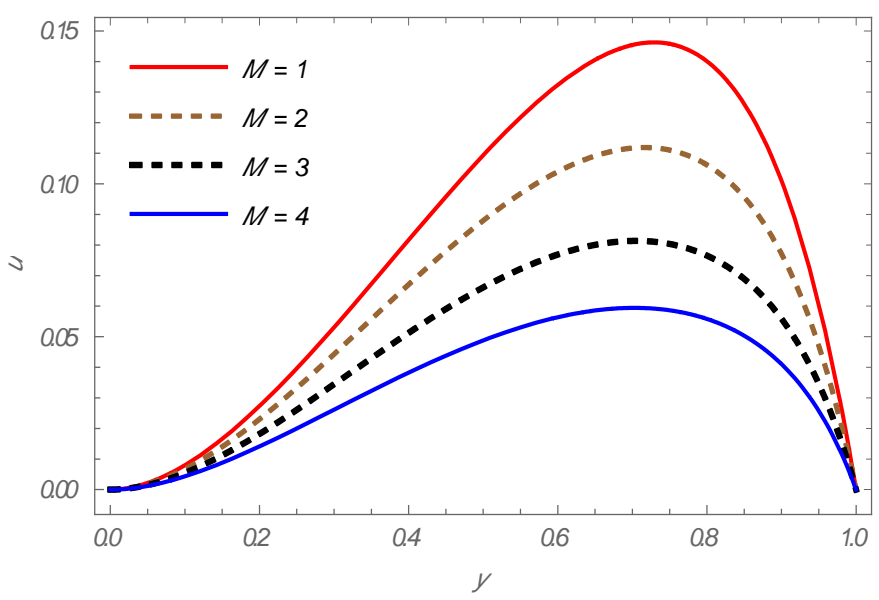

Fig. 7. Figure .7 Velocity profile for $\boldsymbol{M}$ with $D a=1, \mathrm{~W}=1, \lambda=1, \operatorname{Re}=1, \mathrm{~N}=1.25, M=$ $1, P e=2, G r=2, S=0.5, t=0.5$. 


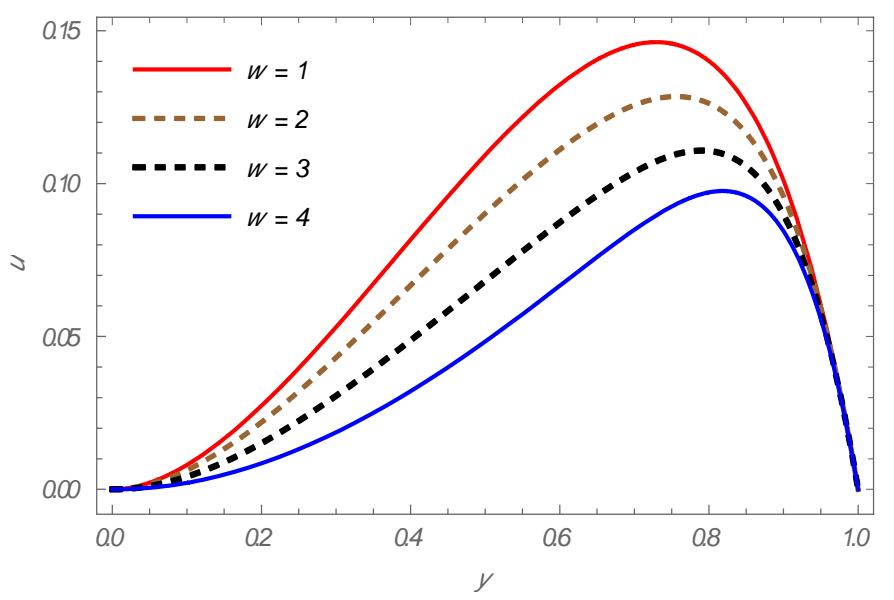

Fig. 8. Velocity profile for $\boldsymbol{W}$ with $D a=1, M=1, \lambda=1, \operatorname{Re}=1, \mathrm{~N}=1.25, M=1, P e=$ $2, G r=2, S=0.5, t=0.5$.

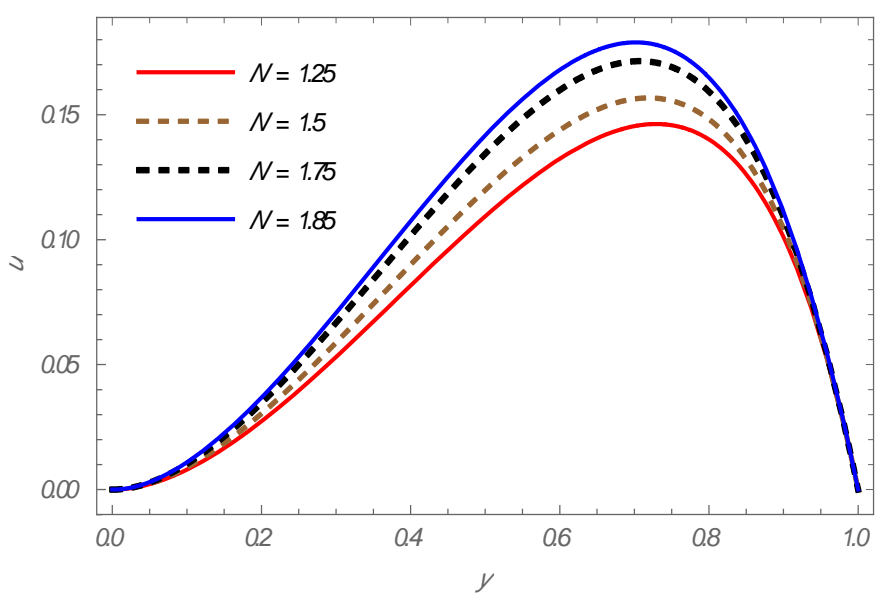

Fig. 9. Velocity profile for $\boldsymbol{N}$ with $D a=1, M=1, \mathrm{~W}=1, \lambda=1, \operatorname{Re}=1, M=1, P e=$ $2, G r=2, S=0.5, t=0.5$. 


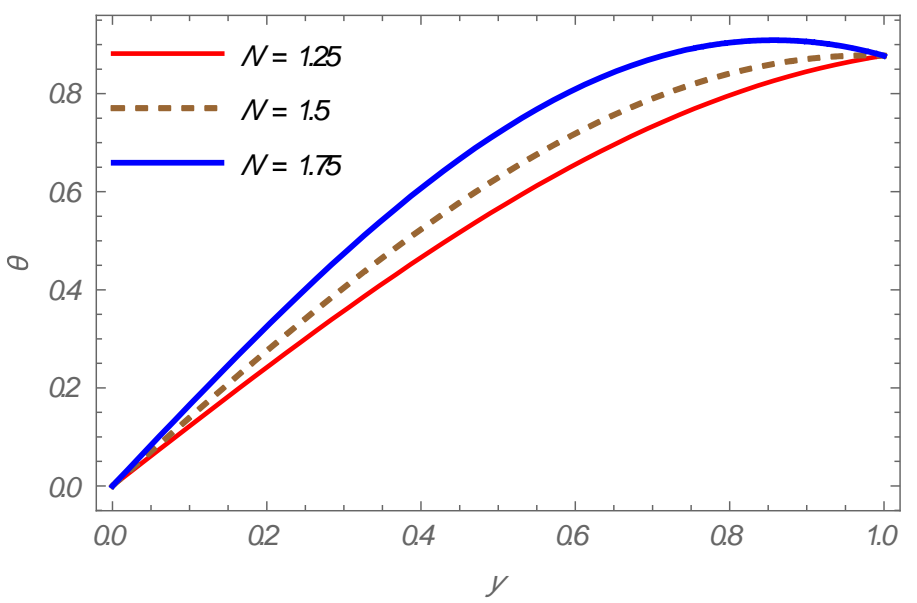

Fig. 10.Influence of $\mathbf{N}$ on Temperature $\boldsymbol{\theta}$ for $\boldsymbol{\omega}=\mathbf{1}, \mathbf{P e}=\mathbf{0 . 7}, \mathbf{t}=\mathbf{0 . 5}$

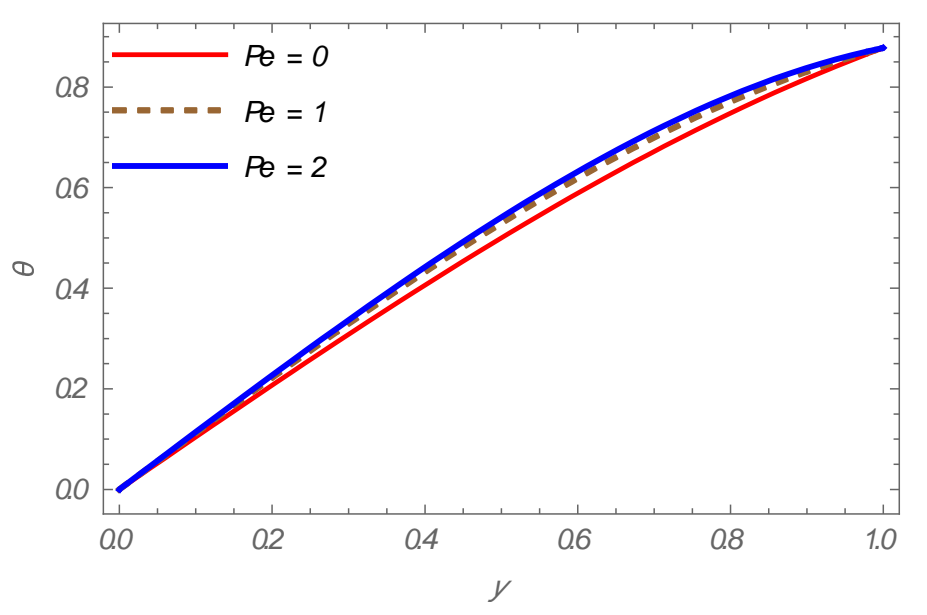

Fig. 11.Influence of Pe on Temperature $\theta$ for $t=0.5, N=1, \omega=1$ 


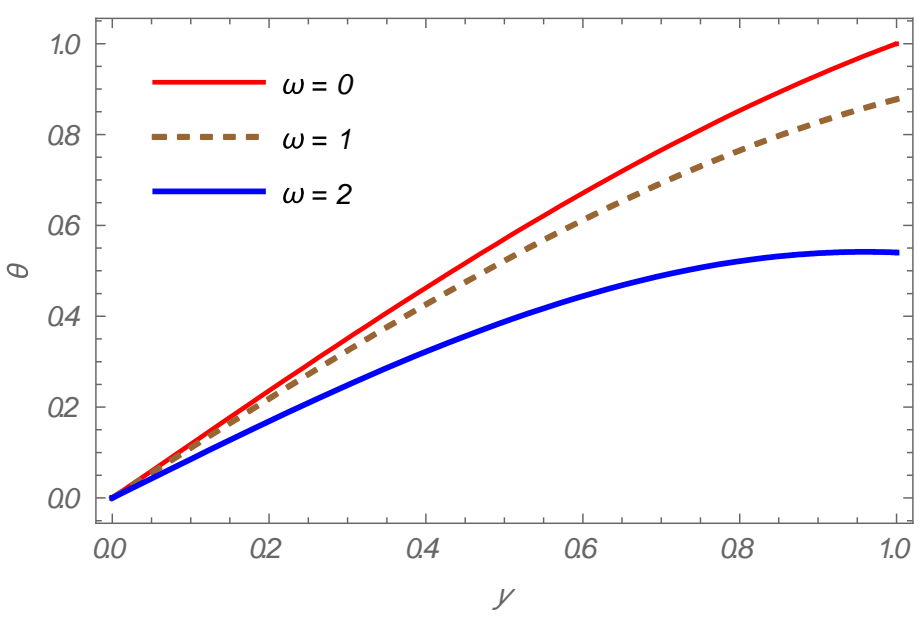

Fig. 12.Influence of $\boldsymbol{\omega}$ on Temperature $\boldsymbol{\theta}$ for $\mathbf{t}=\mathbf{0 . 5}, \mathbf{N}=\mathbf{1}, \mathrm{Pe}=\mathbf{0 . 7}$

\section{Concluding Remarks:}

We discuss the influence of heat transfer on magnetohydrodynamics oscillatory flow of Williamson fluid through a porous medium. The perturbation technique for the two kinds of flow Poiseuille flow and Couette flow. We found the velocity and temperature are analytical. We used different values to finding the results of pertinent parameters, namely Darcy number (Da), Reynolds number (Re), Peclet number (Pe), magnetic parameter $(\mathrm{M})$, Grashof number $(\mathrm{Gr})$, Weissenberg number $(W e)$, frequency of the oscillation $(\omega)$ and radiation parameter $(N)$ for the velocity and temperature.

The key points are:

- The velocity profiles were increased by the increasing $R e, N, D a, \lambda$ and $\mathrm{Gr}$ for both the Poiseuille and Couette flow.

- The velocity profiles decrease with the increasing $\omega$ and $M$ for both the Poiseuille and Couette flow.

- We show that by increasing $N$ and $P e$ the temperature increasing $\theta$ and the temperature $\theta$ decreases with the increasing $\omega$

\section{References}

[1] Nigam S.D. and Singh S.N. 1960. Heat-transfer by laminar flow between parallel plates under the action of transverse magnetic field. Q. J. Mech. Appl. Math., 13: 85-87.

[2] Attia H.A. and Kotb N.A. 1996. MHD flow between two parallel plates with heat transfer. Acta Mechanic, 117: 215-220.

[3] Raptis, A., Massias, C. and Tzivanidis,G. 1982. Hydromagnetic free convection flow during a porous medium between two parallel plates MHD. Phys. Lett., 90A: 288-289.Khudair and Al-Khafajy Iraqi Journal of Science, 2018, Vol. 59, No.1B, pp: 389-397 
[4] Mostafa, A.A. 2009.Thermal radiation effect on unsteady MHD free convection flow past a vertical plate with temperature dependent viscosity. The Canadian journal of chemical engineering, 87(1): 171-181.

[5] Pandey S, Chaube M (2011) Study of wall properties on peristaltic transport of a couple stress fluid. Meccanica 46(6):1319-1330

[6] Hina S, Hayat T, Asghar S, Obaidat S (2012) Peristaltic flow of Maxwell fluid in an asymmetric channel with wall properties. Int J Phys Sci 7(14):2145-2155

[7] Radhakrishnamacharya G, Srinivasulu C (2007) Influence of wall properties on peristaltic transport with heat transfer. CR Mec 335(7):369-373

[8] Srinivas S, Gayathri R, Kothandapani M (2009) The influence of slip conditions, wall properties and heat transfer on MHD peristaltic transport. Comput Phys Commun 180(11):21152122

[9] Nadeem, S., Youssef, M.M., Zehra, I. 2015. Numerical solutions of Williamson fluid with pressure dependent Viscosity, Elsevier Results in Physics,pp.20-25.

[10] Vinvent, W.G., Cogley, A.C.L. and Giles, E.S. 1968. Differential approximation for radiative heat-transfer in non-linear equations-grey gas near equilibrium, American Institute of Aeronautics and Astronautics, pp. 551-553.

Article submitted 1 January 2021. Published as resubmitted by the authors 1 April 2021. 